\title{
Heterosis and Genetic Behavior of Some Yield and Yield Component Traits in Squash (Cucurbita pepo, L.)
}

\author{
A. H. Abd El-Hadi; El-Adl, A. M. ${ }^{1}$; Horeya M. Fathy and M. A. Abdein ${ }^{2}$
}

\begin{abstract}
An 7 x 7 complete diallel cross of squash was evaluated with parents for heterotic manifestation and evaluate the genetic behavior of yield and yield component traits. Seven different squash varieties belong to the species (Cucurbita pepo, L.), were used in this study. These parental varieties were: Eskandarani $\left(P_{1}\right)$; Zucca Patisson custard white $\left(\mathbf{P}_{2}\right)$; All Green Bush $\left(\mathbf{P}_{3}\right)$; Courgette Orelia $\left(\mathbf{P}_{4}\right)$; Sakiz $\left(P_{5}\right)$; Copi $\left(P_{6}\right)$ and Gapla $\left(P_{7}\right)$. The seeds of these parental varieties were obtained from different countries: $\left(P_{1}\right)$ and $\left(P_{6}\right)$ from Egypt; $\left(P_{2}\right)$ from France; $\left(P_{3}\right)$ from United Kingdom (U.K.); $\left(P_{4}\right)$ from Germany; $\left(P_{5}\right)$ from Turkey and $\left(P_{7}\right)$ from Syria. These parental varieties were used and their $42 F_{1,1 r}$ hybrids were obtained through complete diallel crosses mating design system.
\end{abstract}

Data were recorded for seven traits: fruit length (F.L.cm); fruit diameter (F.D.cm); fruit shape index (F.Sh.I.); Total Soluble Solid\% (T.S.S\%); weight of fruit (W.F.g); number of fruits per plant (No.F./P.) and fruit yield per plant (F.Y./P.kg). The results also indicated that the amounts of heterosis versus mid-parents showed highly significant values for all studied traits. The estimates of heterosis versus the better parent showed highly significance for most studied traits. None of the hybrids exhibited maximum heterosis for all the traits, but significant and desirable level of heterosis over midparents and better parent was obtained in several hybrids for the different traits. However, GCA values were larger than their corresponding estimates of SCA for studied yield and yield component traits at both $F_{1,1 r}$ hybrids. Reciprocal effects ( $r$ ) were significant for most studied traits. The results indicated that the parents $\mathbf{P}_{1}, \mathbf{P}_{3}, \mathbf{P}_{4}, \mathbf{P}_{5}$ and $P_{7}$ were seemed to be the best combiners for fruit length (F.L.cm), fruit shape index (F.Sh.I.) and number of fruits per plant (No.F./P.). Also, $P_{2}$ was the best combiner for Total Soluble Solid\% (T.S.S\%). In the same time, the two parents $P_{2}$ and $P_{6}$ were the best combiners for fruit diameter (F.D.cm) and weight of fruit (W.F.g).

These results indicated that the parents $P_{1}, P_{2}$ and $P_{4}$ were seemed to be the best combiners for fruit yield per plant (F.Y./P.kg). All 49 genotypes (seven parents, $21 F_{1}$ 's and 21 reciprocal hybrids) were evaluated in a field trial at the growing summer. The experimental design was the Randomized Complete Blocks Design (RCBD) with three replications of 2010. This study was conducted in the Kaha Research Farm of Vegetables Breeding Department, Horticultural Research Institute, (HRI), Agric. Res. Center (ARC), Giza, Egypt.

\footnotetext{
${ }^{1}$ Dept. of Genetics, Faculty of Agric. Mansoura University, Egypt.

${ }^{2}$ Vegetables Breeding Department, Horticulture Res. Inst. (HRI),

ARC, Giza, Egypt.

E- mail: abdeingene@yahoo.com

Received July 22, 2014, Accepted August 21, 2014
}

Keywords: squash, diallel, yield, yield component, heterosis and genetic parameters.

\section{INTRODUCTION}

The manifestations of heterosis as well as the nature of gene action were studied in squash by many authors among them Gabr (2003), Abd El-Hadi et al., (2004), Abdein (2005), Al-Ballat (2008) and Al-Araby (2010). They estimated heterosis for yield and some economical traits in squash. They found that heterosis was observed of only over the mid-parents but also over its beter parent for all yield and yield component traits. Thangamani, et al., (2011) in bitter gourd studied full diallel analysis was carried out with 10 diversified parents to study the heterosis for yield and quality traits. In this respect, Jahan et al., (2012) in sweet gourd (Cucurbita moschata Duch.ex Poir) found significant and desirable level of mid and better parent heterosis values in the studied hybrids for No. of fruits per plant and fruit yield per plant. Similarly, Marie et al., (2012) indicated that heterosis over mid- parents was evident in all yield traits, where the hybrid (IL3 $\times$ IL6) exhibited (16.89 and $57.57 \%$ ) for the ratio pistilate flower $\%$ and fruit number per plant, respectively.

Concerning general and specific combining abilities, in summer squash, Helmy (1993) evaluated $15 \quad F_{1}$ hybrids and their parents. The obtained results concluded that the best combiner was parent6, followed by parents 7 and 5, respectively, which exhibited the highest positive values of GCA effects for total fruits number and weight. El-Adl et al., (1996) studied the combining ability among six inbred lines of agoor (Cucumis melo var. chata, L.) and regarded that the mean squares of GCA and SCA were highly significant for yield per plant. In this respect, Ana and Staub (2002) found that the combining ability was significantly influenced by year for most of yield traits. In the same time, Feyzian et al., (2009) found that additive gene effects were most important with respect to average weight per fruit and yield, while genetic dominance effects were also important yield. Nahavand and Tashkandi as parents had significant positive general combining ability effects for yield and acceptable yield. Douglas et al., (2011) found additive 
and non-additive gene effects were important in the expression of parthenocarpy and resistance to PRSV-W in summer squash.

Pradip et al., (2013) in ridge gourd [Luffa acutangula (Roxb.) L.] studied 28 genotypes, including seven parental lines and their 21 crosses. The highly significant mean squares due to parents, hybrids and parents versus hybrids; and GCA and SCA for yield and antioxidants (Ascorbic Acid, Total Carotenoids and Total Phenolics) indicated the existence of abundant genetic variation. Recently, Sanin et al., (2014) studied the predominance of additive gene action over the dominance type for the traits under study suggests that a recurrent selection program could serve as a strategy to increase the frequencies of genes that promote the expression of traits associated with seed production and starch content in butternut squash.

Iathet and Piluek (2006) found on melon, that broad heritability of number fruits per plant and plant yield were as high as $(0.60$ and 0.61 , respectively). Fruit number per plant had highly positive correlation to yield per plant. Mishra et al., (2007) observed maximum heritability for yield per plant followed by number of fruits per plant on cucumber.

\section{MATERIALS AND METHODS}

Seven different squash varieties belong to the species (Cucurbita pepo, L.). were used in this study. These parental varieties were: Eskandarani $\left(\mathrm{P}_{1}\right)$; Zucca Patisson custard white $\left(\mathrm{P}_{2}\right)$; All Green Bush $\left(\mathrm{P}_{3}\right)$; Courgette Orelia $\left(\mathrm{P}_{4}\right)$; Sakiz $\left(\mathrm{P}_{5}\right)$; Copi $\left(\mathrm{P}_{6}\right)$ and Gapla $\left(\mathrm{P}_{7}\right)$. The seeds of these parental varieties were obtained from different countries: $\left(\mathrm{P}_{1}\right)$ and $\left(\mathrm{P}_{6}\right)$ from Egypt; $\left(\mathrm{P}_{2}\right)$ from France; $\left(\mathrm{P}_{3}\right)$ from United Kingdom (U.K.); $\left(\mathrm{P}_{4}\right)$ from Germany; $\left(\mathrm{P}_{5}\right)$ from Turkey and $\left(\mathrm{P}_{7}\right)$ from Syria.

All these varieties represented a wide range of variability for most studied yield and yield component traits. Plants from each parental variety were selfpollinated for three successive generations to obtain an inbred from each variety. In the summer season of 2009, all single crosses including reciprocals were made among these seven varieties according to a complete diallel crosses mating design system to produce $21 \mathrm{~F}_{1}$ hybrids and their $21 \mathrm{~F}_{1 \mathrm{r}}$ reciprocal hybrids. In addition, the seven parental varieties were also self-pollinated to obtain enough seeds from each variety. All 49 genotypes (seven parents, $21 \mathrm{~F}_{1}$ 's and 21 reciprocal hybrids) were evaluated in a field trial at the growing summer season of 2010 at Kaha Vegetables Research Station, Kaha, Kalubia, Egypt.

The experimental design was the Randomized Complete Blocks Design (RCBD) with three replications. The plot or the experimental unit was one ridge $5.0 \mathrm{~m}$. long and $1.0 \mathrm{~m}$. wide. The distance between hills $0.5 \mathrm{~m}$. apart. Therefore, each ridge contained 10 hills.

Data were recorded on several randomly chosen plants within each plot for the following traits: fruit length (F.L.cm); fruit diameter (F.D.cm); fruit shape index (F.Sh.I.); Total Soluble Solid\% (T.S.S\%); weight of fruit (W.F.g); number of fruits per plant (No.F./P.) and fruit yield per plant (F.Y./P.kg). The significance of differences among genetic means for all studied traits were tested according to F-test. The analysis of variances and the expectations of mean squares were made according to Steel and Torrie (1960).

The amounts of heterosis were determined as the deviation of the mid-parents and the better parent as follows:

1. Heterosis from the mid-parents:

$$
\mathbf{H}_{1,}^{\left(\mathrm{F}_{1},{ }_{\text {M.P. }} \%\right.} \%=\frac{\left(\mathrm{F}_{1}-\mathrm{M.P}\right)}{---------} \times 100
$$

2. Heterosis from the better parent:

$$
\mathrm{H}_{1,}^{\left(\mathrm{F}_{1}{ }_{\text {B.P. }} \%\right.} \%=\frac{\left(\mathrm{F}_{1}-\text { B.P }\right)}{--------} \times 100
$$

The analysis of variance of diallel crosses were made to obtain the estimates of general combining ability (G.C.A.), specific combining ability (S.C.A.) and reciprocal effect (r). The procedures of these analyses were described by Griffing (1956) method I.

The estimates of GCA variance $\left(\delta^{2} \mathrm{~g}\right)$ and SCA variance $\left(\delta^{2} s\right)$ could be expressed in terms of genetic variances according to Matzingar and Kempthorne (1956) and Cockerham (1963) with the assumption that there was no epistasis into additive and non- additive variances.

\section{RESULTS AND DISCUSSIONS}

\section{Analysis of variances:-}

In this investigation, many yield and yield component traits were studied. These traits included: fruit length (F.L.cm); fruit diameter (F.D.cm); fruit shape index (F.Sh.I.); Total Soluble Solid\% (T.S.S\%); weight of fruit (W.F.g); number of fruits per plant (No.F./P.) and fruit yield per plant (F.Y./P.kg). The analysis of variance and mean squares for yield and yield component traits for all genotypes are presented in Table 1.

The results indicated that the mean squares of all genotypes were highly significant for all yield traits. These results revealed the presence of large variations among the yield and yield component traits. 
Table 1. Analysis of variance and mean squares for yield and yield component traits

\begin{tabular}{ccccccccc}
\hline & & \multicolumn{7}{c}{ Yield and yield component traits } \\
\cline { 3 - 9 } S.V. & d.f. & F.L.cm & F.D.cm & F.Sh.I. & T.S.S.\% & W.F.g & No.F./P. & F.Y./P.kg \\
\hline Reps. & 2 & $2.744^{* *}$ & 0.074 & 0.029 & 0.053 & $63.879^{* *}$ & 0.106 & $0.325^{*}$ \\
\hline Genotypes & 48 & $20.989^{* *}$ & $4.241^{* *}$ & $4.695^{* *}$ & $5.832^{* *}$ & $4841.734^{* *}$ & $168.297^{* *}$ & $2.891^{* *}$ \\
\hline Error & 96 & 0.080 & 0.043 & 0.048 & 0.019 & 12.825 & 0.800 & 0.072 \\
\hline
\end{tabular}

*,** Significant at 0.05 and 0.01 levels of probability, respectively.

These results were expected where the genotypes in this investigation included variable genetic parental varieties, $F_{1}$ hybrids and their $F_{1 r}$ (reciprocal) hybrids. Thus, the partition of the genetic variation to its components could be made through the analysis of the complete diallel crosses mating design system.

\section{The mean performance of all genotypes:}

In this investigation, many yield and yield component traits were studied. The means of yield and yield component traits were obtained for the seven parental varieties, their $\mathrm{F}_{1}, \mathrm{~F}_{1 \mathrm{r}}$ (reciprocal) hybrids and the results are presented in Tables 2, 3 and 4, respectively. The results cleared that the obtained mean values showed that there was no single parent exceeded all the other parents for all yield studied traits.

The results presented in Table 2, showed that the parental variety $\mathrm{P}_{2}$ was the lowest parent for F.L.cm, F.Sh.I. and F.Y./P.kg. In the same time $\mathrm{P}_{2}$ was the highest parent for F.D.cm and T.S.S.\% traits the $\mathrm{P}_{4}$ was the highest parent for F.L.cm, F.Sh.I. and No.F./P. traits. while the parental variety $\mathrm{P}_{7}$ was the highest parent for F.Y./P.kg traits. In the same time, The parental variety $\mathrm{P}_{6}$ was the highest parent for W.F.g trait.

The parental variety $\mathrm{P}_{3}$ had the lowest values for F.D.cm and W.F.g traits. It is also, noticed from the same table that the differences between the means of the lowest and the highest parent were always significant indicating the presence of genetic differences between the seven parental verities were used in this investigation. In general, these results suggested that there was a wide range of variation among the seven parental varieties for all studied traits.

The results are presented in Tables 3 and 4 indicated that the highest $F_{1}$ hybrid for fruit yield per plant was $P_{2}$ $\times \mathrm{P}_{3}$ with the mean value of $5.86 \mathrm{~kg}$. Whereas, the highest $\mathrm{F}_{1 \mathrm{r}}$ (reciprocal) hybrid was $\mathrm{P}_{7} \times \mathrm{P}_{6}$ with the mean of $5.91 \mathrm{~kg}$. On the other hand, $\mathrm{F}_{1}$ hybrid $\mathrm{P}_{5} \times \mathrm{P}_{7}$ was the lowest with the mean value of $3.74 \mathrm{~kg}$. While, $\mathrm{P}_{6} \times \mathrm{P}_{5} \mathrm{~F}_{1 \mathrm{r}}$ (reciprocal) hybrid was the lowest with the mean value of $3.99 \mathrm{~kg}$ for the same trait.

The results showed that the means of the $F_{1}$ hybrids ranged from 8.73 to $16.27 \mathrm{~cm} ; 2.87$ to $5.97 \mathrm{~cm} ; 1.57$ to $4.92 ; 3.62$ to $8.17 ; 117.2$ to $221.2 \mathrm{~g} ; 21.3$ to 43.8 fruits and 3.74 to $5.86 \mathrm{~kg}$ for F.L.cm; F.D.cm; F.Sh.I.; T.S.S.\%; W.F.g; No.F./P. and F.Y./P.kg., respectively. On the other hand, the mean values in the $F_{1 \mathrm{r}}$ (reciprocal) hybrids ranged from 8.83 to $16.63 \mathrm{~cm} ; 3.03$ to $5.73 \mathrm{~cm} ; 1.58$ to $4.94 ; 4.06$ to $7.77 ; 113.3$ to $226.1 \mathrm{~g}$; 23.4 to 44.6 fruits and 3.99 to $5.91 \mathrm{~kg}$ for the same traits, respectively.

Concerning the performances of the $F_{1}$ and $F_{1 r}$ (reciprocal) hybrids for yield and yield component traits, the results indicated that the magnitudes of the means of the $F_{1}$ and their $F_{1 r}$ (reciprocal) hybrids were close to each other for most studied traits.

Table 2. The mean performances of seven parental varieties for yield and yield component traits

\begin{tabular}{|c|c|c|c|c|c|c|c|c|}
\hline \multirow[t]{2}{*}{ No. } & \multirow[t]{2}{*}{ Parents } & \multicolumn{7}{|c|}{ Yield and yield component traits } \\
\hline & & F.L.cm & F.D.cm & F.Sh.I. & T.S.S.\% & W.F.g & No.F./P. & F.Y./P.kg \\
\hline 1 & $\mathrm{P}_{1}$ & 12.93 & 3.13 & 4.24 & 3.33 & 117.6 & 23.4 & 2.73 \\
\hline 2 & $\mathrm{P}_{2}$ & $4.73^{\mathrm{L}}$ & $7.77^{\mathrm{H}}$ & $0.61^{\mathrm{L}}$ & $5.63^{\mathrm{H}}$ & 130.1 & 15.9 & $2.11^{\mathrm{L}}$ \\
\hline 3 & $\mathrm{P}_{3}$ & 10.93 & $2.63^{\mathrm{L}}$ & 4.15 & 3.53 & $93.9^{\mathrm{L}}$ & 24.8 & 2.33 \\
\hline 4 & $\mathrm{P}_{4}$ & $13.07^{\mathrm{H}}$ & 2.83 & $4.61^{\mathrm{H}}$ & 4.37 & 98.9 & $30.2^{\mathrm{H}}$ & 2.87 \\
\hline 5 & $\mathrm{P}_{5}$ & 11.67 & 3.07 & 3.81 & 3.37 & 107.7 & 26.9 & 2.81 \\
\hline 6 & $\mathrm{P}_{6}$ & 8.63 & 6.53 & 1.32 & $2.43^{\mathrm{L}}$ & $134.9^{\mathrm{H}}$ & $15.1^{\mathrm{L}}$ & 2.23 \\
\hline 7 & $\mathrm{P}_{7}$ & 12.47 & 2.93 & 4.26 & 3.57 & 112.7 & 28.2 & $3.15^{\mathrm{H}}$ \\
\hline \multicolumn{2}{|c|}{ L.S.D. ${ }_{0.05}$} & 0.45 & 0.33 & 0.35 & 0.22 & 8.68 & 1.44 & 0.43 \\
\hline \multicolumn{2}{|c|}{ L.S.D. 0.01} & 0.60 & 0.44 & 0.46 & 0.29 & 11.49 & 1.91 & 0.57 \\
\hline
\end{tabular}


Table 3. The mean performances of the $F_{1}$ hybrids for yield and yield component traits

\begin{tabular}{|c|c|c|c|c|c|c|c|c|}
\hline \multirow[t]{2}{*}{ No. } & \multirow[t]{2}{*}{$F_{1}$ hybrids } & \multicolumn{7}{|c|}{ Yield and yield component traits. } \\
\hline & & F.L.cm & F.D.cm & F.Sh.I. & T.S.S.\% & W.F.g & No.F./P. & F.Y./P.kg \\
\hline 1 & $\mathrm{P}_{1} \times \mathrm{P}_{2}$ & 9.27 & 5.77 & 1.61 & 6.13 & 200.1 & 25.2 & 4.94 \\
\hline 2 & $\mathrm{P}_{1} \times \mathrm{P}_{3}$ & 13.93 & 3.27 & 4.27 & 4.23 & 121.7 & 37.4 & 4.34 \\
\hline 3 & $\mathrm{P}_{1} \times \mathrm{P}_{4}$ & 15.67 & 3.43 & 4.56 & 5.17 & 123.1 & 43.1 & 5.26 \\
\hline 4 & $\mathrm{P}_{1} \times \mathrm{P}_{5}$ & 14.53 & 3.53 & 4.12 & 4.47 & 125.4 & 41.1 & 4.71 \\
\hline 5 & $\mathrm{P}_{1} \times \mathrm{P}_{6}$ & 13.43 & $2.87^{\mathrm{L}}$ & 4.71 & $3.62^{\mathrm{L}}$ & 208.1 & 28.2 & 5.79 \\
\hline 6 & $\mathrm{P}_{1} \times \mathrm{P}_{7}$ & $16.27^{\mathrm{H}}$ & 3.43 & 4.74 & 4.27 & 120.2 & 39.2 & 4.55 \\
\hline 7 & $\mathrm{P}_{2} \times \mathrm{P}_{3}$ & 9.67 & 5.53 & 1.75 & 7.57 & 213.7 & 28.1 & $5.86^{\mathrm{H}}$ \\
\hline 8 & $\mathrm{P}_{2} \times \mathrm{P}_{4}$ & 11.77 & 4.63 & 2.55 & $8.17^{\mathrm{H}}$ & 176.7 & 32.8 & 5.17 \\
\hline 9 & $\mathrm{P}_{2} \times \mathrm{P}_{5}$ & 9.37 & 5.73 & 1.64 & 7.33 & 188.2 & 28.4 & 4.77 \\
\hline 10 & $\mathrm{P}_{2} \times \mathrm{P}_{6}$ & 8.97 & $5.97^{\mathrm{H}}$ & 1.51 & 6.47 & $221.2^{\mathrm{H}}$ & $21.3^{\mathrm{L}}$ & 4.65 \\
\hline 11 & $\mathrm{P}_{2} \times \mathrm{P}_{7}$ & $8.73^{\mathrm{L}}$ & 5.57 & $1.57^{\mathrm{L}}$ & 7.43 & 171.8 & 28.6 & 4.55 \\
\hline 12 & $\mathrm{P}_{3} \times \mathrm{P}_{4}$ & 16.27 & 3.33 & 4.88 & 4.43 & $117.2^{\mathrm{L}}$ & 42.9 & 4.83 \\
\hline 13 & $\mathrm{P}_{3} \times \mathrm{P}_{5}$ & 15.73 & 3.27 & 4.82 & 4.77 & 124.2 & 39.2 & 4.79 \\
\hline 14 & $\mathrm{P}_{3} \times \mathrm{P}_{6}$ & 14.83 & 4.33 & 3.42 & 3.83 & 198.2 & 28.6 & 4.87 \\
\hline 15 & $\mathrm{P}_{3} \times \mathrm{P}_{7}$ & 14.07 & 3.27 & 4.32 & 4.33 & 117.6 & 39.7 & 4.57 \\
\hline 16 & $\mathrm{P}_{4} \times \mathrm{P}_{5}$ & 15.93 & 3.37 & 4.74 & 6.27 & 126.2 & $43.8^{\mathrm{H}}$ & 5.47 \\
\hline 17 & $\mathrm{P}_{4} \times \mathrm{P}_{6}$ & 12.73 & 3.07 & 4.16 & 5.27 & 137.8 & 32.3 & 4.51 \\
\hline 18 & $\mathrm{P}_{4} \times \mathrm{P}_{7}$ & 14.43 & 2.97 & $4.92^{\mathrm{H}}$ & 5.77 & 119.9 & 41.9 & 4.88 \\
\hline 19 & $\mathrm{P}_{5} \times \mathrm{P}_{6}$ & 13.73 & 3.67 & 3.75 & 4.73 & 154.2 & 28.1 & 4.32 \\
\hline 20 & $\mathrm{P}_{5} \times \mathrm{P}_{7}$ & 15.53 & 3.27 & 4.76 & 5.47 & 118.9 & 29.7 & $3.74^{\mathrm{L}}$ \\
\hline 21 & $\mathrm{P}_{6} \times \mathrm{P}_{7}$ & 14.67 & 5.17 & 2.84 & 4.07 & 149.3 & 29.2 & 4.28 \\
\hline & L.S.D. 0.05 & 0.45 & 0.33 & 0.35 & 0.22 & 8.68 & 1.44 & 0.43 \\
\hline & L.S.D. ${ }_{0.01}$ & 0.60 & 0.44 & 0.46 & 0.29 & 11.49 & 1.91 & 0.57 \\
\hline
\end{tabular}

$\mathrm{H}=$ The highest value.

$\mathrm{L}=$ The lowest value.

Table 4. The mean performances of the $21 \mathrm{~F}_{1 \mathrm{r}}$ hybrids for yield and yield component traits

\begin{tabular}{|c|c|c|c|c|c|c|c|c|}
\hline \multirow[t]{2}{*}{ No. } & \multirow[t]{2}{*}{$F_{1 \mathrm{r}}$ hybrids } & \multicolumn{7}{|c|}{ Yield and yield component traits } \\
\hline & & F.L.cm & F.D.cm & F.Sh.I. & T.S.S.\% & W.F.g & No.F./P. & F.Y./P.kg \\
\hline 22 & $\mathrm{P}_{2} \times \mathrm{P}_{1}$ & 8.90 & 5.63 & $1.58^{\mathrm{L}}$ & $7.77^{\mathrm{H}}$ & 193.6 & 27.6 & 5.34 \\
\hline 23 & $\mathrm{P}_{3} \times \mathrm{P}_{1}$ & 13.73 & 3.07 & 4.52 & 4.53 & 123.2 & 41.1 & 5.04 \\
\hline 24 & $\mathrm{P}_{3} \times \mathrm{P}_{2}$ & 10.27 & $5.73^{\mathrm{H}}$ & 1.79 & 7.37 & 208.8 & 28.2 & 5.77 \\
\hline 25 & $\mathrm{P}_{4} \times \mathrm{P}_{1}$ & 15.77 & 3.27 & 4.83 & 6.23 & 125.2 & 39.8 & 4.93 \\
\hline 26 & $\mathrm{P}_{4} \times \mathrm{P}_{2}$ & 12.67 & 3.53 & 3.59 & 8.07 & 186.6 & 32.1 & 5.23 \\
\hline 27 & $\mathrm{P}_{4} \times \mathrm{P}_{3}$ & $16.63^{\mathrm{H}}$ & 3.37 & $4.94^{\mathrm{H}}$ & 5.37 & 117.1 & 38.7 & 4.55 \\
\hline 28 & $\mathrm{P}_{5} \times \mathrm{P}_{1}$ & 14.77 & 3.27 & 4.56 & 5.57 & 125.7 & 40.9 & 5.01 \\
\hline 29 & $\mathrm{P}_{5} \times \mathrm{P}_{2}$ & $8.83^{L}$ & 5.47 & 1.62 & 6.87 & 215.3 & 27.6 & 5.76 \\
\hline 30 & $\mathrm{P}_{5} \times \mathrm{P}_{3}$ & 12.67 & $3.03^{\mathrm{L}}$ & 4.19 & 5.07 & $113.3^{\mathrm{L}}$ & 38.9 & 4.43 \\
\hline 31 & $\mathrm{P}_{5} \times \mathrm{P}_{4}$ & 13.47 & 3.13 & 4.33 & 6.37 & 126.1 & 41.3 & 5.32 \\
\hline 32 & $\mathrm{P}_{6} \times \mathrm{P}_{1}$ & 14.13 & 4.37 & 3.24 & $4.06^{\mathrm{L}}$ & 192.8 & 27.1 & 5.22 \\
\hline 33 & $\mathrm{P}_{6} \times \mathrm{P}_{2}$ & 12.13 & 4.57 & 2.67 & 5.73 & $226.1^{\mathrm{H}}$ & $23.4^{\mathrm{L}}$ & 4.91 \\
\hline 34 & $\mathrm{P}_{6} \times \mathrm{P}_{3}$ & 14.27 & 4.43 & 3.22 & 4.57 & 152.3 & 29.2 & 4.41 \\
\hline 35 & $\mathrm{P}_{6} \times \mathrm{P}_{4}$ & 13.77 & 3.53 & 3.91 & 5.23 & 216.7 & 31.5 & 5.87 \\
\hline 36 & $\mathrm{P}_{6} \times \mathrm{P}_{5}$ & 12.23 & 4.27 & 2.87 & 4.33 & 135.7 & 29.5 & $3.99^{\mathrm{L}}$ \\
\hline 37 & $\mathrm{P}_{7} \times \mathrm{P}_{1}$ & 15.73 & 3.27 & 4.82 & 4.67 & 120.8 & $44.6^{\mathrm{H}}$ & 5.38 \\
\hline 38 & $\mathrm{P}_{7} \times \mathrm{P}_{2}$ & 9.37 & 5.33 & 1.76 & 7.47 & 211.1 & 29.4 & 5.73 \\
\hline 39 & $\mathrm{P}_{7} \times \mathrm{P}_{3}$ & 14.63 & 3.47 & 4.22 & 5.67 & 114.8 & 36.7 & 4.26 \\
\hline 40 & $\mathrm{P}_{7} \times \mathrm{P}_{4}$ & 15.17 & 3.33 & 4.55 & 6.53 & 118.1 & 42.9 & 5.04 \\
\hline 41 & $\mathrm{P}_{7} \times \mathrm{P}_{5}$ & 14.67 & 3.23 & 4.54 & 5.43 & 128.1 & 41.7 & 5.31 \\
\hline 42 & $\mathrm{P}_{7} \times \mathrm{P}_{6}$ & 14.23 & 5.17 & 2.76 & 4.77 & 204.7 & 29.1 & $5.91^{\mathrm{H}}$ \\
\hline & L.S.D.0.05 & 0.45 & 0.33 & 0.35 & 0.22 & 8.68 & 1.44 & 0.43 \\
\hline & L.S.D.0.01 & 0.60 & 0.44 & 0.46 & 0.29 & 11.49 & 1.91 & 0.57 \\
\hline
\end{tabular}


At the same time, when hybrids were compared with each other, the results showed the presence of significant differences between them for many yield traits. It was obtained that some $\mathrm{F}_{1}$ and $\mathrm{F}_{1 \mathrm{r}}$ (reciprocal) hybrids for yield and yield component traits exceeded the better parent and naturally exceed versus the midparents.

\section{Heterosis:-}

\subsection{Heterosis from the mid-parents $\left(\mathrm{H}_{\text {M.P. } \%):-}\right.$}

Heterosis percentage of the $42 \mathrm{~F}_{1,1 \mathrm{r}}$ hybrids relative to the mid-parents for the yield and yield component traits are presented in Tables 5 and 6 .

The results indicated that the values of heterosis over the mid-parents for $F_{1}$ hybrids ranged from 1.55 to $51.62 \%$ for F.L.cm; -40.69 to $21.95 \%$ for F.D.cm; 35.51 to $69.34 \%$ for F.Sh.I.; 12.24 to $65.09 \%$ for T.S.S.\%; 4.33 to $90.86 \%$ for W.F.g.; 8.06 to $63.55 \%$ for No.F./P. and 25.6 to $163.9 \%$ for F.Y./P.kg. With the respect to heterosis from mid-parents $\left(\mathrm{H}_{\text {M.P. }} \%\right)$ for $\mathrm{F}_{1}$ hybrids. The results reveled that $19 ; 9 ; 11 ; 21 ; 20 ; 21$ and
21 out of the $21 \mathrm{~F}_{1}$ hybrids and 20;6;10;21;20;21 and 21 $\mathrm{F}_{1 \mathrm{r}}$ hybrids out of the $21 \mathrm{~F}_{1 \mathrm{r}}$ hybrids exhibited highly significant desirable heterosis values for F.L.cm; F.D.cm; F.Sh.I.; T.S.S.\%; W.F.g; No.F./P. and F.Y./P.kg, respectively.

While, the values of heterosis over the mid-parents for $\mathrm{F}_{1 \mathrm{r}}$ (reciprocal) hybrids ranged from 0.75 to $81.55 \%$; -36.13 to $24.55 \%$; -34.67 to $176.02 \% ; 32.04$ to $73.23 \%$; 4.85 to $86.43 \% ; 29.17$ to $72.74 \%$ and 55.5 to $159.8 \%$ for F.L.cm; F.D.cm; F.Sh.I.; T.S.S.\%; W.F.g; No.F./P. and F.Y./P.kg, respectively.

Similar results were obtained by Abd El-Hadi et al., (2001); Abd El-Hadi et al., (2004); Iathet and Piluek (2006); Al-Araby, (2010); Thangamani et al., (2011); Jahan et al., (2012) and Marie et al., (2012).

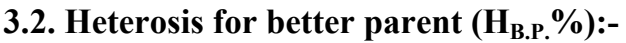

Heterosis percentage of the $42 \mathrm{~F}_{1}$ hybrids relative to the better parent for the yield and yield component traits are presented in Tables 7 and 8 .

Table 5. Heterosis percentage reltive to the mid-parents of the $F_{1}$ hybrids for yield and yield component traits

\begin{tabular}{|c|c|c|c|c|c|c|c|c|}
\hline \multirow[b]{2}{*}{ No. } & \multirow{2}{*}{$\begin{array}{c}F_{1} \\
\text { hybrids }\end{array}$} & \multicolumn{7}{|c|}{ Yield and yield component traits. } \\
\hline & & F.L.cm & F.D.cm & F.Sh.I. & T.S.S.\% & W.F.g & No.F./P. & F.Y./P.kg \\
\hline 1 & $\mathrm{P}_{1} \times \mathrm{P}_{2}$ & $4.91 *$ & $5.81 *$ & $-33.68 * *$ & $36.80 * *$ & $61.52 * *$ & $28.07 * *$ & $104.3 * *$ \\
\hline 2 & $\mathrm{P}_{1} \times \mathrm{P}_{3}$ & $16.76 * *$ & $13.29 * *$ & 1.77 & $23.30 * *$ & $15.05 * *$ & $55.08 * *$ & $71.3 * *$ \\
\hline 3 & $\mathrm{P}_{1} \times \mathrm{P}_{4}$ & $20.51 * *$ & $15.08 * *$ & 3.15 & $34.20 * *$ & $13.71 * *$ & $60.50 * *$ & $87.7 * *$ \\
\hline 4 & $\mathrm{P}_{1} \times \mathrm{P}_{5}$ & $18.16^{* *}$ & $13.98 * *$ & 2.42 & $33.33 * *$ & $11.30 * *$ & $63.55 * * \mathrm{H}$ & $69.9 * *$ \\
\hline 5 & $\mathrm{P}_{1} \times \mathrm{P}_{6}$ & $24.57 * *$ & $-40.69 * * \mathrm{~L}$ & $69.34 * * \mathrm{H}$ & $25.66^{* *}$ & $64.79 * *$ & $46.32 * *$ & $133.4 * *$ \\
\hline 6 & $\mathrm{P}_{1} \times \mathrm{P}_{7}$ & $28.08 * *$ & $13.19 * *$ & $11.67 * *$ & $23.67 * *$ & $4.33 * \mathrm{~L}$ & $51.94 * *$ & $54.8 * *$ \\
\hline 7 & $\mathrm{P}_{2} \times \mathrm{P}_{3}$ & $23.40 * *$ & $6.41^{*}$ & $-26.63 * *$ & $65.09 * * \mathrm{H}$ & $90.86^{* * \mathrm{H}}$ & $38.20^{* *}$ & $163.9 * * \mathrm{H}$ \\
\hline 8 & $\mathrm{P}_{2} \times \mathrm{P}_{4}$ & $32.21 * *$ & $-12.58 * *$ & -2.37 & $63.33 * *$ & $54.30 * *$ & $42.30 * *$ & $107.5^{* *}$ \\
\hline 9 & $\mathrm{P}_{2} \times \mathrm{P}_{5}$ & $14.23 * *$ & $5.85 *$ & $-26.06 * *$ & $62.96 * *$ & $58.28 * *$ & $33.07 * *$ & $93.8 * *$ \\
\hline 10 & $\mathrm{P}_{2} \times \mathrm{P}_{6}$ & $34.16 * *$ & $-16.55 * *$ & $55.95 * *$ & $60.33 * *$ & $66.90 * *$ & $37.72 * *$ & $113.9^{* *}$ \\
\hline 11 & $\mathrm{P}_{2} \times \mathrm{P}_{7}$ & $1.55^{\mathrm{L}}$ & 4.05 & $-35.51 * \mathrm{~L}$ & $61.59 * *$ & $41.52 * *$ & $29.75^{* *}$ & $73.1 * *$ \\
\hline 12 & $\mathrm{P}_{3} \times \mathrm{P}_{4}$ & $35.56 * *$ & $21.95 * * \mathrm{H}$ & $11.38^{* *}$ & $12.24 * * \mathrm{~L}$ & $21.54 * *$ & $55.78^{* *}$ & $85.5^{* *}$ \\
\hline 13 & $\mathrm{P}_{3} \times \mathrm{P}_{5}$ & $39.23 * *$ & $14.62 * *$ & $21.03 * *$ & $38.16^{* *}$ & $23.23 * *$ & $51.61 * *$ & $86.5 * *$ \\
\hline 14 & $\mathrm{P}_{3} \times \mathrm{P}_{6}$ & $51.62 * * \mathrm{H}$ & $-5.45^{*}$ & $25.10 * *$ & $28.49 * *$ & $73.25 * *$ & $43.31 * *$ & $113.2 * *$ \\
\hline 15 & $\mathrm{P}_{3} \times \mathrm{P}_{7}$ & $20.23^{* *}$ & $17.37 * *$ & 2.82 & $22.07 * *$ & $13.81 * *$ & $49.78 * *$ & $66.7 * *$ \\
\hline 16 & $\mathrm{P}_{4} \times \mathrm{P}_{5}$ & $28.84 * *$ & $14.12 * *$ & $12.53 * *$ & $62.07 * *$ & $22.15 * *$ & $53.30 * *$ & $92.7 * *$ \\
\hline 17 & $\mathrm{P}_{4} \times \mathrm{P}_{6}$ & $17.36^{* *}$ & $-34.52 * *$ & $40.21 * *$ & $54.90 * *$ & $17.87^{* *}$ & $42.46^{* *}$ & $76.7 * *$ \\
\hline 18 & $\mathrm{P}_{4} \times \mathrm{P}_{7}$ & $13.05 * *$ & 2.89 & $10.87 * *$ & $45.38 * *$ & $13.32 * *$ & $43.61 * *$ & $62.1 * *$ \\
\hline 19 & $\mathrm{P}_{5} \times \mathrm{P}_{6}$ & $35.30 * *$ & $-23.61 * *$ & $45.81^{* *}$ & $63.22 * *$ & $27.11 * *$ & $34.18^{* *}$ & $71.4^{* *}$ \\
\hline 20 & $\mathrm{P}_{5} \times \mathrm{P}_{7}$ & $28.73 * *$ & $8.89 *$ & $17.82 * *$ & $57.69 * *$ & $7.91 * *$ & $8.06 * * \mathrm{~L}$ & $25.6^{* * \mathrm{~L}}$ \\
\hline 21 & $\mathrm{P}_{6} \times \mathrm{P}_{7}$ & $39.02 * *$ & $9.15 * *$ & 1.89 & $35.56 * *$ & $20.59 * *$ & $34.93 * *$ & $58.9 * *$ \\
\hline \multicolumn{2}{|c|}{ L.S.D..$_{0.05}$} & 0.34 & 0.25 & 0.26 & 0.16 & 4.37 & 1.09 & 0.32 \\
\hline \multicolumn{2}{|c|}{ L.S.D. ${ }_{0.01}$} & 0.45 & 0.33 & 0.35 & 0.22 & 5.76 & 1.44 & 0.43 \\
\hline
\end{tabular}

*,** Significant and highly significant at 0.05 and 0.01 probability levels, respectively. 
Table 6. Heterosis percentage reltive of the mid-parents of $F_{1 r}$ hybrids for yield and yield component traits

\begin{tabular}{|c|c|c|c|c|c|c|c|c|}
\hline \multirow[b]{2}{*}{ No. } & \multirow{2}{*}{$\begin{array}{c}F_{1 r} \\
\text { hybrids }\end{array}$} & \multicolumn{7}{|c|}{ Yield and yield component traits. } \\
\hline & & F.L.cm & F.D.cm & F.Sh.I. & T.S.S.\% & W.F.g & No.F./P. & F.Y./P.kg \\
\hline 22 & $\mathrm{P}_{2} \times \mathrm{P}_{1}$ & $0.75^{\mathrm{L}}$ & 3.36 & $-34.67 * * \mathrm{~L}$ & $73.23 * * \mathrm{H}$ & $56.27 * *$ & $40.63 * *$ & $120.5 * *$ \\
\hline 23 & $\mathrm{P}_{3} \times \mathrm{P}_{1}$ & $15.08 * *$ & 6.36 & $7.72 *$ & $32.04 * * \mathrm{~L}$ & $16.50 * *$ & $70.42 * *$ & $99.4 * *$ \\
\hline 24 & $\mathrm{P}_{3} \times \mathrm{P}_{2}$ & $31.06 * *$ & $10.26 * *$ & $-24.74 * *$ & $60.73 * *$ & $86.43 * * \mathrm{H}$ & $38.69 * *$ & $159.8 * * \mathrm{H}$ \\
\hline 25 & $\mathrm{P}_{4} \times \mathrm{P}_{1}$ & $21.28 * *$ & $9.50 * *$ & $9.25 * *$ & $61.90 * *$ & $15.59 * *$ & $48.32 * *$ & $76.2 * *$ \\
\hline 26 & $\mathrm{P}_{4} \times \mathrm{P}_{2}$ & $42.32 * *$ & $-33.33 * *$ & $37.35 * *$ & $61.33 * *$ & $62.92 * *$ & $39.41 * *$ & $110.1 * *$ \\
\hline 27 & $\mathrm{P}_{4} \times \mathrm{P}_{3}$ & $38.61 * *$ & $23.17 * *$ & $12.74 * *$ & $35.86 * *$ & $21.44 * *$ & $40.76 * *$ & $75.1 * *$ \\
\hline 28 & $\mathrm{P}_{5} \times \mathrm{P}_{1}$ & $20.05 * *$ & 5.38 & $13.40 * *$ & $66.17 * *$ & $11.57 * *$ & $62.76 * *$ & $81.1 * *$ \\
\hline 29 & $\mathrm{P}_{5} \times \mathrm{P}_{2}$ & $7.72 * *$ & 0.92 & $-26.98 * *$ & $52.59 * *$ & $81.05 * *$ & $29.17 * * \mathrm{~L}$ & $134.3 * *$ \\
\hline 30 & $\mathrm{P}_{5} \times \mathrm{P}_{3}$ & $12.09 * *$ & 6.43 & 5.21 & $46.86 * *$ & $12.45 * *$ & $50.45 * *$ & $72.4 * *$ \\
\hline 31 & $\mathrm{P}_{5} \times \mathrm{P}_{4}$ & $8.89 * *$ & 6.21 & 2.70 & $64.66 * *$ & $22.08 * *$ & $44.78 * *$ & $87.3 * *$ \\
\hline 32 & $\mathrm{P}_{6} \times \mathrm{P}_{1}$ & $31.07 * *$ & $-9.66^{* *}$ & $16.54 * *$ & $40.92 * *$ & $52.65^{* *}$ & $40.61 * *$ & $110.2 * *$ \\
\hline 33 & $\mathrm{P}_{6} \times \mathrm{P}_{2}$ & $81.55 * * \mathrm{H}$ & $-36.13 * * \mathrm{~L}$ & $176.02 * * \mathrm{H}$ & $42.15 * *$ & $70.65 * *$ & $51.51 * *$ & $125.8 * *$ \\
\hline 34 & $\mathrm{P}_{6} \times \mathrm{P}_{3}$ & $45.83 * *$ & -3.27 & $17.68 * *$ & $53.07 * *$ & $33.10 * *$ & $46.66 * *$ & $93.4 * *$ \\
\hline 35 & $\mathrm{P}_{6} \times \mathrm{P}_{4}$ & $26.88 * *$ & $-24.56 * *$ & $31.69 * *$ & $53.92 * *$ & $85.35 * *$ & $39.07 * *$ & $130.1 * *$ \\
\hline 36 & $\mathrm{P}_{6} \times \mathrm{P}_{5}$ & $20.53 * *$ & $-11.11 * *$ & $11.57 *$ & $49.43 * *$ & $11.83 * *$ & $40.70 * *$ & $58.4 * *$ \\
\hline 37 & $\mathrm{P}_{7} \times \mathrm{P}_{1}$ & $23.88 * *$ & 7.69 & $13.47 * *$ & $35.27 * *$ & $4.85 * \mathrm{~L}$ & $72.74 * * \mathrm{H}$ & $83.1 * *$ \\
\hline 38 & $\mathrm{P}_{7} \times \mathrm{P}_{2}$ & $8.91 * *$ & -0.31 & $-27.82 * *$ & $62.32 * *$ & $73.89 * *$ & $33.54 * *$ & $117.9 * *$ \\
\hline 39 & $\mathrm{P}_{7} \times \mathrm{P}_{3}$ & $25.07 * *$ & $24.55 * * \mathrm{H}$ & 0.43 & $59.62 * *$ & $11.10 * *$ & $38.70 * *$ & $55.5 * * \mathrm{~L}$ \\
\hline 40 & $\mathrm{P}_{7} \times \mathrm{P}_{4}$ & $18.80^{* *}$ & $15.61 * *$ & 2.70 & $64.71 * *$ & $11.59 * *$ & $46.92 * *$ & $67.6^{* *}$ \\
\hline 41 & $\mathrm{P}_{7} \times \mathrm{P}_{5}$ & $21.55^{* *}$ & 7.78 & $12.42 * *$ & $56.73 * *$ & $16.26 * *$ & $51.42 * *$ & $78.2 * *$ \\
\hline 42 & $\mathrm{P}_{7} \times \mathrm{P}_{6}$ & $34.91 * *$ & $9.15 * *$ & -1.24 & $58.89 * *$ & $65.30 * *$ & $34.46 * *$ & $119.5 * *$ \\
\hline & D.D.0.05 & 0.34 & 0.25 & 0.26 & 0.16 & 4.37 & 1.09 & 0.32 \\
\hline & . . $_{0.01}$ & 0.45 & 0.33 & 0.35 & 0.22 & 5.76 & 1.44 & 0.43 \\
\hline
\end{tabular}

*,** Significant and highly significant at 0.05 and 0.01 probability levels, respectively.

$\mathrm{H}=$ The highest value

$\mathrm{L}=$ The lowest value

Table 7. Heterosis better parent of $F_{1}$ hybrids for yield and yield component traits

\begin{tabular}{|c|c|c|c|c|c|c|c|c|}
\hline \multirow[t]{2}{*}{ No. } & \multirow{2}{*}{$\begin{array}{c}F_{1} \\
\text { hybrids }\end{array}$} & \multicolumn{7}{|c|}{ Yield and yield component traits. } \\
\hline & & F.L.cm & F.D.cm & F.Sh.I. & T.S.S.\% & W.F.g & No.F./P. & F.Y./P.kg \\
\hline 1 & $\mathrm{P}_{1} \times \mathrm{P}_{2}$ & $-28.35 * *$ & $-25.75^{*} *$ & $-62.06 * * \mathrm{~L}$ & 8.88 & 53.78 & 7.40 & 81.2 \\
\hline 2 & $\mathrm{P}_{1} \times \mathrm{P}_{3}$ & $7.73 * *$ & 4.26 & 0.76 & 19.81 & 3.43 & 50.81 & 58.8 \\
\hline 3 & $\mathrm{P}_{1} \times \mathrm{P}_{4}$ & $19.90 * *$ & 9.57 & -1.06 & 18.32 & 4.68 & 42.45 & 83.1 \\
\hline 4 & $\mathrm{P}_{1} \times \mathrm{P}_{5}$ & $12.37 * *$ & $12.77^{*}$ & -2.67 & 32.67 & 6.60 & $53.10^{\mathrm{H}}$ & 67.6 \\
\hline 5 & $\mathrm{P}_{1} \times \mathrm{P}_{6}$ & $3.87 *$ & $-56.12 * * \mathrm{~L}$ & $11.10 * *$ & 8.70 & 54.22 & 20.20 & 112.3 \\
\hline 6 & $\mathrm{P}_{1} \times \mathrm{P}_{7}$ & $25.77 * *$ & 9.57 & $11.40 * *$ & 19.63 & $2.15^{\mathrm{L}}$ & 39.17 & 44.5 \\
\hline 7 & $\mathrm{P}_{2} \times \mathrm{P}_{3}$ & $-11.59 * *$ & $-28.76^{* *}$ & $-57.92 * *$ & 34.32 & $64.28^{\mathrm{H}}$ & 13.31 & $151.5^{\mathrm{H}}$ \\
\hline 8 & $\mathrm{P}_{2} \times \mathrm{P}_{4}$ & $-9.95 * *$ & $-40.34 * *$ & $-44.72 * *$ & 44.97 & 35.82 & 8.49 & 80.1 \\
\hline 9 & $\mathrm{P}_{2} \times \mathrm{P}_{5}$ & $-19.71 * *$ & $-26.18 * *$ & $-57.11^{* *}$ & 30.18 & 44.66 & 5.83 & 69.8 \\
\hline 10 & $\mathrm{P}_{2} \times \mathrm{P}_{6}$ & 3.86 & $-23.18 * *$ & $13.97^{\mathrm{H}}$ & 14.79 & 63.91 & 34.24 & 108.1 \\
\hline 11 & $\mathrm{P}_{2} \times \mathrm{P}_{7}$ & $-29.95 * * \mathrm{~L}$ & $-28.33 * *$ & $-63.13 * *$ & 31.95 & 32.08 & $1.42^{\mathrm{L}}$ & 44.7 \\
\hline 12 & $\mathrm{P}_{3} \times \mathrm{P}_{4}$ & $24.49 * *$ & $17.65 *^{\mathrm{H}}$ & 5.82 & $1.53^{\mathrm{L}}$ & 18.43 & 41.79 & 68.1 \\
\hline 13 & $\mathrm{P}_{3} \times \mathrm{P}_{5}$ & $34.86^{* *}$ & 6.52 & $16.12 * *$ & 34.91 & 15.32 & 45.78 & 70.7 \\
\hline 14 & $\mathrm{P}_{3} \times \mathrm{P}_{6}$ & $35.67 * * \mathrm{H}$ & $-33.67 * *$ & $-17.53 * *$ & 8.49 & 46.89 & 15.19 & 108.8 \\
\hline 15 & $\mathrm{P}_{3} \times \mathrm{P}_{7}$ & $12.83^{* *}$ & $11.36^{*}$ & 1.55 & 21.50 & 4.29 & 40.83 & 45.1 \\
\hline 16 & $\mathrm{P}_{4} \times \mathrm{P}_{5}$ & $21.94 * *$ & 9.78 & 2.79 & 43.51 & 17.18 & 44.76 & 90.6 \\
\hline 17 & $\mathrm{P}_{4} \times \mathrm{P}_{6}$ & -2.55 & $-53.06 * *$ & $-9.80 *$ & 20.61 & $2.15^{L}$ & 6.73 & 57.1 \\
\hline 18 & $\mathrm{P}_{4} \times \mathrm{P}_{7}$ & $10.46^{* *}$ & 1.14 & 6.59 & 32.06 & 6.39 & 38.70 & 54.9 \\
\hline 19 & $\mathrm{P}_{5} \times \mathrm{P}_{6}$ & $17.71 * *$ & $-43.88 * *$ & -1.82 & 40.59 & 14.28 & 4.71 & 53.9 \\
\hline 20 & $\mathrm{P}_{5} \times \mathrm{P}_{7}$ & $24.60 * *$ & 6.52 & $11.71 * *$ & $53.27^{\mathrm{H}}$ & 5.50 & 5.56 & $18.8^{\mathrm{L}}$ \\
\hline 21 & $\mathrm{P}_{6} \times \mathrm{P}_{7}$ & $17.65 * *$ & $-20.92 * *$ & $-33.23 * *$ & 14.02 & 10.67 & 3.55 & 35.9 \\
\hline L.S.I & & 0.45 & 0.33 & 0.35 & 0.22 & 8.68 & 1.44 & 0.43 \\
\hline L.S.I & & 0.60 & 0.44 & 0.46 & 0.29 & 11.49 & 1.91 & 0.57 \\
\hline
\end{tabular}


*** Significant and highly significant at 0.05 and 0.01 probability levels, respectively.

$\mathrm{H}=$ The highest value.

$\mathrm{L}=$ The lowest value. 
Table 8. Hetrosis better parent of $F_{1 r}$ hybrids for yield and yield component traits

\begin{tabular}{|c|c|c|c|c|c|c|c|c|}
\hline \multirow[b]{2}{*}{ No. } & \multirow{2}{*}{$\begin{array}{c}F_{1 r} \\
\text { hybrids }\end{array}$} & \multicolumn{7}{|c|}{ Yield and yield component traits. } \\
\hline & & F.L.cm & F.D.cm & F.Sh.I. & T.S.S.\% & W.F.g & No.F./P. & F.Y./P.kg \\
\hline 22 & $\mathrm{P}_{2} \times \mathrm{P}_{1}$ & $-31.19 * * \mathrm{~L}$ & $-27.47 * *$ & $-62.63 * * \mathrm{~L}$ & 37.87 & 48.78 & 17.92 & 95.6 \\
\hline 23 & $\mathrm{P}_{3} \times \mathrm{P}_{1}$ & $6.19 * *$ & -2.13 & 6.66 & 28.30 & 4.73 & $65.73^{\mathrm{H}}$ & 84.9 \\
\hline 24 & $\mathrm{P}_{3} \times \mathrm{P}_{2}$ & $-6.10 * *$ & $-26.18 * *$ & $-56.84 * *$ & 30.77 & 60.47 & 13.71 & $147.6^{\mathrm{H}}$ \\
\hline 25 & $\mathrm{P}_{4} \times \mathrm{P}_{1}$ & $20.66 * *$ & 4.26 & 4.79 & 42.75 & 6.40 & 31.64 & 71.9 \\
\hline 26 & $\mathrm{P}_{4} \times \mathrm{P}_{2}$ & -3.06 & $-54.51 * * \mathrm{~L}$ & $-22.24 * *$ & 43.20 & 43.40 & 6.28 & 82.4 \\
\hline 27 & $\mathrm{P}_{4} \times \mathrm{P}_{3}$ & $27.30 * *$ & $18.82 * * \mathrm{H}$ & $7.11 *$ & 22.90 & 18.33 & 28.11 & 58.7 \\
\hline 28 & $\mathrm{P}_{5} \times \mathrm{P}_{1}$ & $14.18 * *$ & 4.26 & $7.76^{*}$ & $65.35^{\mathrm{H}}$ & 6.86 & 52.36 & 78.5 \\
\hline 29 & $\mathrm{P}_{5} \times \mathrm{P}_{2}$ & $-24.29 * *$ & $-29.61 * *$ & $-57.65 * *$ & 21.89 & 65.46 & $2.73^{\mathrm{L}}$ & 105.3 \\
\hline 30 & $\mathrm{P}_{5} \times \mathrm{P}_{3}$ & $8.57 * *$ & -1.09 & 0.94 & 43.40 & 5.23 & 44.67 & 57.8 \\
\hline 31 & $\mathrm{P}_{5} \times \mathrm{P}_{4}$ & 3.06 & 2.17 & -6.18 & 45.80 & 17.12 & 36.71 & 85.3 \\
\hline 32 & $\mathrm{P}_{6} \times \mathrm{P}_{1}$ & $9.28 * *$ & $-33.16 * *$ & $-23.54 * *$ & 21.90 & 42.86 & 15.50 & 91.1 \\
\hline 33 & $\mathrm{P}_{6} \times \mathrm{P}_{2}$ & $40.54 * * \mathrm{H}$ & $-41.20 * *$ & $101.72 * * \mathrm{H}$ & $1.78^{\mathrm{L}}$ & $67.59^{\mathrm{H}}$ & 47.69 & 119.6 \\
\hline 34 & $\mathrm{P}_{6} \times \mathrm{P}_{3}$ & $30.49 * *$ & $-32.14 * *$ & $-22.42 * *$ & 29.25 & 12.85 & 17.88 & 89.4 \\
\hline 35 & $\mathrm{P}_{6} \times \mathrm{P}_{4}$ & $5.36^{* *}$ & $-45.92 * *$ & $-15.28 * *$ & 19.85 & 60.62 & 4.19 & 104.6 \\
\hline 36 & $\mathrm{P}_{6} \times \mathrm{P}_{5}$ & $4.86^{*}$ & $-34.69 * *$ & $-24.88 * *$ & 28.71 & $0.54^{\mathrm{L}}$ & 9.80 & 42.2 \\
\hline 37 & $\mathrm{P}_{7} \times \mathrm{P}_{1}$ & $21.65 * *$ & 4.26 & $13.19 * *$ & 30.84 & 2.66 & 58.22 & 70.9 \\
\hline 38 & $\mathrm{P}_{7} \times \mathrm{P}_{2}$ & $-24.87 * *$ & $-31.33 * *$ & $-58.74 * *$ & 32.54 & 62.29 & 4.38 & 82.1 \\
\hline 39 & $\mathrm{P}_{7} \times \mathrm{P}_{3}$ & $17.38 * *$ & $18.18 * *$ & -0.81 & 58.88 & 1.80 & 30.41 & $35.3^{\mathrm{L}}$ \\
\hline 40 & $\mathrm{P}_{7} \times \mathrm{P}_{4}$ & $16.07 * *$ & $13.64 * * \backslash$ & -1.27 & 49.62 & 4.76 & 41.90 & 60.2 \\
\hline 41 & $\mathrm{P}_{7} \times \mathrm{P}_{5}$ & $17.65^{* *}$ & 5.43 & 6.58 & 52.34 & 13.66 & 47.93 & 68.6 \\
\hline 42 & $\mathrm{P}_{7} \times \mathrm{P}_{6}$ & $14.17 * *$ & $-20.92 * *$ & $-35.28 * *$ & 33.64 & 51.70 & 3.20 & 87.6 \\
\hline & S.D. ${ }_{0.05}$ & 0.45 & 0.33 & 0.35 & 0.22 & 8.68 & 1.44 & 0.43 \\
\hline & S.D. 0.01 & 0.60 & 0.44 & 0.46 & 0.29 & 11.49 & 1.91 & 0.57 \\
\hline
\end{tabular}

*** Significant and highly significant at 0.05 and 0.01 probability levels, respectively.

$\mathrm{H}=$ The highest value.

The results indicated that the values of heterosis over the better parent for $\mathrm{F}_{1}$ hybrids ranged from -29.95 to $35.67 \%$ for F.L.cm; -56.12 to $17.65 \%$ for F.D.cm; 62.06 to $13.97 \%$ for F.Sh.I.; 1.53 to $53.27 \%$ for T.S.S. $\%$; 2.15 to $64.28 \%$ for W.F.g.; 1.42 to $53.10 \%$ for No.F./P. and 18.8 to $151.5 \%$ for F.Y./P.kg. While, the values of heterosis over the better parent for $F_{1 r}$ (reciprocal) hybrids ranged from -31.19 to $40.54 \%$; 54.51 to $18.82 \%$; -62.63 to $101.72 \% ; 1.78$ to $65.35 \%$; 0.54 to $67.59 \% ; 2.73$ to $65.73 \%$ and 35.3 to $147.6 \%$ for F.L.cm; F.D.cm; F.Sh.I.; T.S.S.\%; W.F.g; No.F./P. and F.Y./P.kg., respectively.

With the respect to heterosis from better parent $\left(\mathrm{H}_{\text {M.P. }} \%\right.$ ) for $\mathrm{F}_{1}$ hybrids. the results reveled that 12 and 5 out of the $21 \mathrm{~F}_{1}$ hybrids exhibited highly significant desirable heterosis values for F.L.cm and F.Sh.I. and 14,3 and $2 F_{1 r}$ hybrids out of the $21 F_{1 r}$ hybrids exhibited highly significant desirable heterosis values for F.L.cm; F.D.cm and F.Sh.I., respectively.

These results were in agreement with the results obtained by Abd El-Hadi et al., (2001); Abd El-Hadi et al., (2004); Iathet and Piluek (2006); Al-Araby, (2010);
Thangamani, et al., (2011); Jahan et al., (2012) and Marie et al., (2012).

\section{Analysis of combining ability variances:}

The variance for combining ability of the seven parental varieties and their $42 \mathrm{~F}_{1}$, $\mathrm{r}$ hybrids for yield and yield component traits are presented in Table 9. The results revealed that the mean squares due to crosses were highly significant for all studied traits. The mean squares due to general combining ability (GCA) exhibited significant differences for all studied traits. The mean squares due to specific combining ability (SCA) were highly significant for all studied traits. It means that the additive genetic variance was more important in the inheritance of these yield traits. At the same time the mean squares due to reciprocal effect were highly significant for all studied traits.

The values of GCA mean squares were higher than those of SCA mean squares for all yield studied traits except for F.Y./P.kg. It means that additive genetic variances were more important in the inheritance of these traits. The present results are in agreement in the reported by Ana and Staub (2002); Kamooh (2002); AlBallat (2008); Feyzian et al., (2009); Pradip et al., (2013) and Sanin et al., (2014). 
Table 9. Analysis of combining abilities and mean squares of the $F_{1}$ hybrids for yield and yield component traits

\begin{tabular}{|c|c|c|c|c|c|c|c|c|}
\hline \multirow[b]{2}{*}{ S.V. } & \multirow[b]{2}{*}{ d.f. } & \multicolumn{7}{|c|}{ Yield and yield component traits } \\
\hline & & F.L.cm & F.D.cm & F.Sh.I & T.S.S.\% & W.F.g & No.F./P. & F.Y./P.kg \\
\hline Reps. & 2 & $0.325^{*}$ & 0.106 & $63.879 * *$ & 0.053 & 0.029 & 0.074 & $2.744 * *$ \\
\hline Crosses & 41 & $2.184 * *$ & $0.347 * *$ & $0.274 * *$ & $0.865 * *$ & $803.395 * *$ & $24.198 * *$ & $1.125 * *$ \\
\hline G.C.A. & 6 & $41.230 * *$ & $10.342 * *$ & $10.641 * *$ & $9.800 * *$ & $7435.883 * *$ & $281.484 * *$ & $0.312 * *$ \\
\hline S.C.A. & 14 & $3.545 * *$ & $0.550 * *$ & $0.394 * *$ & $1.263 * *$ & $1220.231 * *$ & $41.501 * *$ & $1.884 * *$ \\
\hline R.E. & 21 & $0.720 * *$ & $0.127 * *$ & $0.143 * *$ & $0.427 * *$ & $348.693 * *$ & $5.743 * *$ & $0.312 * *$ \\
\hline Error & 82 & 0.08 & 0.043 & 0.048 & 0.019 & 12.825 & 0.800 & 0.072 \\
\hline $\begin{array}{l}\text { G.C.A./ } \\
\text { S.C.A. }\end{array}$ & & 0.848 & 1.451 & 2.187 & 0.562 & 0.439 & 0.493 & 0.009 \\
\hline
\end{tabular}

*** Significant at 0.05 and 0.01 levels of probability, respectively.

\section{Genetic parameters and heritability:}

The relative magnitudes of genetic parameters were estimated for yield and yield component traits and the obtained results are shown in Table 10. The results showed that both additive $\left(\delta^{2} \mathrm{~A}\right)$ and non-additive genetic variances including dominance $\left(\delta^{2} \mathrm{D}\right)$ were positive for all studied yield and yield component traits except $\delta^{2}$ A for F.Y./P.kg which were this indicated that these variances play a role in genetic expression of yield and yield component traits. It was found that the magnitudes of additive genetic variance were larger than dominance genetic variances for all studied traits except for F.Y./P.kg trait. Thus, it could be suggest that additive genetic variance predominated in the inheritance of these yield studied traits. The results also illustrated the importance of reciprocal variances which were smaller than additive genetic variances. Therefore, the cytoplasmic genetic factors also would contribute to the genetic expression of yield and yield component traits. In general, the heritability in broad sense $\left(\mathrm{h}_{\mathrm{b}}^{2} \%\right)$ were higher and larger than their corresponding values of heritability in narrow sense $\left(\mathrm{h}^{2} \mathrm{n} \%\right)$ for all studied traits. The estimated values of heritability in broad sense $\left(\mathrm{h}_{\mathrm{b}}^{2} \%\right)$ ranged from $99.006 \%$ for F.L.cm, $97.636 \%$ for
F.D.cm, 97.277\% for F.Sh.I., 99.128\% for T.S.S, $99.283 \%$ for W.F.g, $98.703 \%$ for No.F./P and $94.348 \%$ for F.Y./P.kg. In the same time, the highest values of $\mathrm{h}^{2} \mathrm{n} \%$ were $82.926 \%$ for F.Sh.I. followed by $78.72 \%$ for F.D.cm and $69.316 \%$ for F.L.cm. These results are according with the results obtained by Al-Ballat (2008); Al-Araby, (2010); Pradip et al., (2013) and Sanin et al., (2014).

6. General combining ability effects $\left(g_{i}\right)$ for the parents:

The general combining ability effects $\left(g_{i}\right)$ of the seven parents for yield and yield component traits are presented in Table 11.

The results revealed that the GCA effects $\left(\mathrm{g}_{\mathrm{i}}\right)$ were computed to be positive and highly significant for desirable parents No. $\mathrm{P}_{1}, \mathrm{P}_{3}, \mathrm{P}_{4}, \mathrm{P}_{5}$ and $\mathrm{P}_{7}$ for F.L.cm; F.Sh.I. and No.F./P. The results revealed that the GCA effects $\left(g_{i}\right)$ were computed to be positive and highly significant for desirable parent No. $\mathrm{P}_{2}$ for F.D.cm; T.S.S.\% and W.F.g. At the same time, the results revealed that the GCA effects showed undesirable negative and highly significant values for the same parent No. $P_{2}$ for F.L.cm; F.Sh.I. and No.F./P.

Table 10. The relative magnitudes of the different genetic parameters and heritability for yield and yield component traits

\begin{tabular}{cccccccc}
\hline $\begin{array}{c}\text { Genetic } \\
\text { parameters and } \\
\text { heritability }\end{array}$ & F.L.cm & F.D.cm & F.Sh.I & T.S.S.\% & W.F.g & No.F./P. & F.Y./P.kg \\
\hline$\delta^{2} \mathrm{~A}$ & 5.580 & 1.432 & 1.462 & 1.214 & 887.9 & 34.134 & -0.231 \\
\hline$\delta^{2} \mathrm{D}$ & 2.070 & 0.302 & 0.206 & 0.743 & 721.49 & 24.321 & 1.082 \\
\hline$\delta^{2} \mathrm{r}$ & 0.32 & 0.042 & 0.047 & 0.204 & 167.93 & 2.471 & 0.12 \\
\hline$\delta^{2} \mathrm{E}$ & 0.08 & 0.043 & 0.048 & 0.019 & 12.825 & 0.800 & 0.072 \\
\hline $\mathrm{h}^{2} \mathrm{~b} \%$ & 99.006 & 97.636 & 97.277 & 99.128 & 99.283 & 98.703 & 94.348 \\
\hline $\mathrm{h}^{2} \mathrm{n} \%$ & 69.316 & 78.724 & 82.926 & 55.688 & 49.599 & 55.299 & 16.875 \\
\hline
\end{tabular}

Note: Negative values were considered equal to zero during the calculation of heritability in broad and narrow senses. 
Table 11. General combining ability effects $\left(g_{i}\right)$ of the seven parents for yield and yield component traits

\begin{tabular}{cccccccc}
\hline \multirow{2}{*}{ Parents } & \multicolumn{7}{c}{ Yield and yield component traits } \\
\cline { 2 - 7 } & F.L.cm & F.D.cm & F.Sh.I. & T.S.S.\% & W.F.g & No.F./P. & F.Y./P.kg \\
\hline $\mathrm{P}_{1}$ & $0.894^{* *}$ & $-0.408^{* *}$ & $0.505^{* *}$ & $-0.559^{* *}$ & $-6.935^{* *}$ & $1.870^{* *}$ & $0.142^{*}$ \\
\hline $\mathrm{P}_{2}$ & $-3.724^{* *}$ & $1.711^{* *}$ & $-1.738^{* *}$ & $1.678^{* *}$ & $40.278^{* *}$ & $-6.438^{* *}$ & $0.166^{*}$ \\
\hline $\mathrm{P}_{3}$ & $0.704^{* *}$ & $-0.336^{* *}$ & $0.382^{* *}$ & $-0.350^{* *}$ & $-14.316^{* *}$ & $1.632^{* *}$ & -0.119 \\
\hline $\mathrm{P}_{4}$ & $1.251^{* *}$ & $-0.789^{* *}$ & $0.794^{* *}$ & $0.297^{* *}$ & $-15.816^{* *}$ & $4.894^{* *}$ & $0.142^{*}$ \\
\hline $\mathrm{P}_{5}$ & $0.299^{* *}$ & $-0.408^{* *}$ & $0.283^{* *}$ & $-0.131^{* *}$ & $-15.125^{* *}$ & $2.085^{* *}$ & -0.119 \\
\hline $\mathrm{P}_{6}$ & $-0.295^{* *}$ & $0.568^{* *}$ & $-0.560^{* *}$ & $-0.916^{* *}$ & $25.469^{* *}$ & $-6.295^{* *}$ & $-0.190^{* *}$ \\
\hline $\mathrm{P}_{7}$ & $0.870^{* *}$ & $-0.336^{* *}$ & $0.332^{* *}$ & -0.017 & $-13.554^{* *}$ & $2.251^{* *}$ & -0.023 \\
\hline L.S.D(gi).0.05 & 0.138 & 0.101 & 0.107 & 0.067 & 1.754 & 0.438 & 0.131 \\
\hline L.S.D(gi). 0.01 & 0.182 & 0.133 & 0.141 & 0.089 & 2.31 & 0.577 & 0.173 \\
\hline
\end{tabular}

*,** Significant and highly significant at 0.05 and 0.01 probability levels, respectively.

While, the GCA effects were found to be and positive highly significant for desirable parent No. $\mathrm{P}_{6}$ only for F.D.cm and W.F.g. While, the parents No. $\mathrm{P}_{1}, \mathrm{P}_{2}$ and $\mathrm{P}_{4}$ were computed to be positive and desirably significant for F.Y./P.kg.

These results indicated that the parents No. $\mathrm{P}_{1}, \mathrm{P}_{3}$, $\mathrm{P}_{4}, \mathrm{P}_{5}$ and $\mathrm{P}_{7}$ were seemed to be the best combiners for F.L.cm; F.Sh.I. and No.F./P. Also, the parent No. $\mathrm{P}_{2}$ was the best combiner for T.S.S.\%. In the same time, the two parents $\mathrm{P}_{2}$ and $\mathrm{P}_{6}$ were the best combiner for F.D.cm

Table 12. Specific combining ability effects $\left(s_{i j}\right)$ of the $21 F_{1}$ hybrids for yield and yield component traits

\begin{tabular}{|c|c|c|c|c|c|c|c|}
\hline \multirow[b]{2}{*}{$F_{1}$ hybrids } & \multicolumn{7}{|c|}{ Yield and yield component traits } \\
\hline & F.L.cm & F.D.cm & F.Sh.I & T.S.S.\% & W.F.g & No.F./P. & F.Y./P.kg \\
\hline $\mathrm{P}_{1} \times \mathrm{P}_{2}$ & $-1.037 * *$ & 0.289 & $-0.691 * *$ & $0.488 * *$ & $12.72 * *$ & $-1.537^{*}$ & 0.238 \\
\hline $\mathrm{P}_{1} \times \mathrm{P}_{3}$ & 0.034 & -0.163 & 0.091 & 0.017 & $-7.18^{*}$ & $3.224 * *$ & -0.142 \\
\hline $\mathrm{P}_{1} \times \mathrm{P}_{4}$ & $0.653 * *$ & $0.456^{*}$ & -0.178 & $0.369 * *$ & -3.68 & $1.963 *$ & 0.095 \\
\hline $\mathrm{P}_{1} \times \mathrm{P}_{5}$ & $0.772 * *$ & $0.408 *$ & -0.020 & $0.631 * *$ & -2.37 & $4.605 * *$ & 0.357 \\
\hline $\mathrm{P}_{1} \times \mathrm{P}_{6}$ & 0.201 & $-0.568 * *$ & $0.461^{*}$ & -0.083 & $31.23^{* *}$ & -0.680 & $0.928 * *$ \\
\hline $\mathrm{P}_{1} \times \mathrm{P}_{7}$ & $1.201 * *$ & -0.163 & $0.405^{*}$ & -0.149 & $-9.78 * *$ & $5.105 * *$ & 0.428 \\
\hline $\mathrm{P}_{2} \times \mathrm{P}_{3}$ & 0.153 & $0.551 * *$ & $-0.380 *$ & $0.779 * *$ & $34.61 * *$ & 0.534 & $1.333 * *$ \\
\hline $\mathrm{P}_{2} \times \mathrm{P}_{4}$ & $1.605 * *$ & $-0.830 * *$ & $0.480 *$ & $0.631 * *$ & 6.44* & $1.605^{*}$ & 0.238 \\
\hline $\mathrm{P}_{2} \times \mathrm{P}_{5}$ & -0.442 & $0.456^{*}$ & $-0.416^{*}$ & 0.226 & $26.08 * *$ & -0.252 & $0.500 *$ \\
\hline $\mathrm{P}_{2} \times \mathrm{P}_{6}$ & $1.653 * *$ & $-1.020 * *$ & $0.863 * *$ & 0.178 & $7.32 *$ & $2.796^{* *}$ & 0.238 \\
\hline $\mathrm{P}_{2} \times \mathrm{P}_{7}$ & $-1.014 * *$ & 0.051 & $-0.451 *$ & $0.445 * *$ & $13.84 * *$ & 0.582 & 0.404 \\
\hline $\mathrm{P}_{3} \times \mathrm{P}_{4}$ & $1.344 * *$ & 0.051 & 0.163 & $-0.506^{* *}$ & -3.47 & $1.534 *$ & 0.190 \\
\hline $\mathrm{P}_{3} \times \mathrm{P}_{5}$ & $0.629 *$ & -0.330 & $0.424 *$ & 0.088 & -2.49 & $3.010 * *$ & 0.285 \\
\hline $\mathrm{P}_{3} \times \mathrm{P}_{6}$ & $1.224 * *$ & 0.194 & -0.021 & $0.374 * *$ & $13.24 * *$ & 0.891 & 0.357 \\
\hline $\mathrm{P}_{3} \times \mathrm{P}_{7}$ & 0.058 & 0.099 & -0.033 & -0.061 & $-6.56^{*}$ & $1.677 *$ & 0.023 \\
\hline $\mathrm{P}_{4} \times \mathrm{P}_{5}$ & 0.082 & 0.289 & -0.110 & $0.440 * *$ & $6.34 *$ & $3.248 * *$ & $0.690 * *$ \\
\hline $\mathrm{P}_{4} \times \mathrm{P}_{6}$ & $-0.656^{* *}$ & $-0.520 * *$ & 0.172 & 0.226 & $16.91 * *$ & 0.963 & 0.428 \\
\hline $\mathrm{P}_{4} \times \mathrm{P}_{7}$ & $-0.490 *$ & 0.051 & 0.027 & $0.826^{* *}$ & -2.40 & $2.748 * *$ & 0.261 \\
\hline $\mathrm{P}_{5} \times \mathrm{P}_{6}$ & -0.037 & -0.235 & 0.078 & 0.155 & $-16.11 * *$ & 0.439 & -0.309 \\
\hline $\mathrm{P}_{5} \times \mathrm{P}_{7}$ & $0.963 * *$ & -0.330 & $0.471 *$ & $0.255^{*}$ & 1.41 & -1.276 & -0.142 \\
\hline $\mathrm{P}_{6} \times \mathrm{P}_{7}$ & $1.058 * *$ & $0.694 * *$ & $-0.499 * *$ & 0.041 & $14.48^{* *}$ & 0.605 & $0.595^{*}$ \\
\hline L.S.D. $\left(\mathrm{s}_{\mathrm{ij}}\right)_{0.05}$ & 0.480 & 0.351 & 0.371 & 0.233 & 6.077 & 1.517 & 0.455 \\
\hline L.S.D. $\left(\mathrm{s}_{\mathrm{ij}}\right)_{0.01}$ & 0.632 & 0.463 & 0.492 & 0.308 & 8.011 & 2.000 & 0.600 \\
\hline
\end{tabular}

and W.F.g. These results indicated that the parents No. $\mathrm{P}_{1}, \mathrm{P}_{2}$ and $\mathrm{P}_{4}$ seemed to be the best combiners for F.Y.P.kg. These results were in agreement with the results obtained by Abd El-Hadi et al., (2004); Abdein (2005); Al-Ballat (2008); Pradip et al., (2013) and Sanin et al., (2014).

\section{Specific combining ability effects $\left(s_{\mathrm{ij}}\right)$ :}

Estimates of specific combining ability effects $\left(\mathrm{s}_{\mathrm{ij}}\right)$ of the $42 \mathrm{~F}_{1}$, lr hybrids for yield and yield components traits are presented in Tables 12 and 13. 
*,** Significant and highly significant at 0.05 and 0.01 probability levels, respectively .

Table 13. Specific combining ability effects $\left(s_{i j}\right)$ of the $21 F_{1 r}$ hybrids for yield and yield component traits

\begin{tabular}{|c|c|c|c|c|c|c|c|}
\hline \multirow[b]{2}{*}{$F_{1 \mathbf{r}}$ hybrids } & \multicolumn{7}{|c|}{ Yield and yield component traits } \\
\hline & F.L.cm & F.D.cm & F.Sh.I & T.S.S.\% & W.F.g & No.F./P. & F.Y./P.kg \\
\hline $\mathrm{P}_{2} \times \mathrm{P}_{1}$ & 0.167 & $0.333 *$ & 0.026 & $-1.00 * *$ & 3.17 & $-1.500^{*}$ & -0.167 \\
\hline $\mathrm{P}_{3} \times \mathrm{P}_{1}$ & -0.333 & -0.166 & -0.280 & $-0.500 * *$ & -1.00 & $-1.667 * *$ & $-0.500 * *$ \\
\hline $\mathrm{P}_{3} \times \mathrm{P}_{2}$ & -0.167 & 0.001 & -0.021 & 0.167 & 2.67 & 0.001 & 0.001 \\
\hline $\mathrm{P}_{4} \times \mathrm{P}_{1}$ & 0.167 & 0.001 & -0.113 & $-0.500 * *$ & -1.000 & $1.667 * *$ & 0.001 \\
\hline $\mathrm{P}_{4} \times \mathrm{P}_{2}$ & $-0.500^{*}$ & 0.166 & $-0.498 * *$ & 0.001 & -5.000 & 0.333 & -0.167 \\
\hline $\mathrm{P}_{4} \times \mathrm{P}_{3}$ & -0.333 & 0.001 & -0.041 & $-0.500 * *$ & 0.171 & $2.000 * *$ & -0.167 \\
\hline $\mathrm{P}_{5} \times \mathrm{P}_{1}$ & 0.001 & 0.001 & -0.190 & $-0.667 * *$ & 1.000 & 0.167 & 0.001 \\
\hline $\mathrm{P}_{5} \times \mathrm{P}_{2}$ & 0.167 & 0.166 & 0.006 & 0.167 & $-13.67 * *$ & 0.333 & $-0.500 * *$ \\
\hline $\mathrm{P}_{5} \times \mathrm{P}_{3}$ & $1.000 * *$ & 0.001 & 0.125 & 0.0001 & $5.17 *$ & 0.001 & 0.333 \\
\hline $\mathrm{P}_{5} \times \mathrm{P}_{4}$ & $1.333 * *$ & 0.166 & 0.183 & 0.0001 & 0.173 & 1.167 & 0.001 \\
\hline $\mathrm{P}_{6} \times \mathrm{P}_{1}$ & $-0.500^{*}$ & $-0.666 * *$ & $0.698 * *$ & -0.167 & $7.50 * *$ & 0.500 & $0.500 * *$ \\
\hline $\mathrm{P}_{6} \times \mathrm{P}_{2}$ & $-1.667 * *$ & $0.666^{* *}$ & $-0.570 * *$ & $0.333 * *$ & -2.500 & -1.000 & 0.167 \\
\hline $\mathrm{P}_{6} \times \mathrm{P}_{3}$ & 0.333 & -0.166 & 0.105 & $-0.500 * *$ & $23.17 * *$ & -0.500 & 0.333 \\
\hline $\mathrm{P}_{6} \times \mathrm{P}_{4}$ & -0.333 & $-0.333 *$ & 0.031 & 0.001 & $-39.33 * *$ & 0.500 & $-0.667 * *$ \\
\hline $\mathrm{P}_{6} \times \mathrm{P}_{5}$ & $0.667 * *$ & 0.001 & $0.433 * *$ & $0.500 * *$ & $9.33 * *$ & -0.500 & 0.001 \\
\hline $\mathrm{P}_{7} \times \mathrm{P}_{1}$ & 0.333 & 0.166 & -0.031 & $-0.333 * *$ & -0.175 & $-2.500 * *$ & -0.167 \\
\hline $\mathrm{P}_{7} \times \mathrm{P}_{2}$ & -0.167 & 0.166 & -0.088 & -0.167 & $-19.67 * *$ & -0.333 & $-0.500 * *$ \\
\hline $\mathrm{P}_{7} \times \mathrm{P}_{3}$ & -0.333 & -0.166 & 0.036 & $-0.963 * *$ & 1.332 & $1.500 *$ & 0.167 \\
\hline $\mathrm{P}_{7} \times \mathrm{P}_{4}$ & -0.333 & 0.001 & 0.186 & $-0.500 * *$ & 1.000 & -0.500 & 0.001 \\
\hline $\mathrm{P}_{7} \times \mathrm{P}_{5}$ & 0.167 & 0.001 & 0.100 & 0.167 & -4.834 & $-6.000 * *$ & $-0.667 * *$ \\
\hline $\mathrm{P}_{7} \times \mathrm{P}_{6}$ & 0.333 & 0.001 & 0.048 & $-0.500 * *$ & $-27.50 * *$ & 0.167 & $-1.001 * *$ \\
\hline L.S.D. $\left(\mathrm{r}_{\mathrm{ij}}\right)_{0.05}$ & 0.396 & 0.290 & 0.306 & 0.192 & 5.013 & 1.252 & 0.375 \\
\hline L.S.D. $\left(\mathrm{r}_{\mathrm{ij}}\right)_{0.01}$ & 0.522 & 0.382 & 0.404 & 0.253 & 6.609 & 1.650 & 0.495 \\
\hline
\end{tabular}

*,** Significant and highly significant at 0.05 and 0.01 probability levels, respectively .

The $\mathrm{F}_{1}$ hybrids $\mathrm{P}_{2} \times \mathrm{P}_{4}$ and $\mathrm{P}_{2} \times \mathrm{P}_{6}$ showed highly significant positive values of 1.605 and 1.653 for F.L.cm, respectively. While, the $\mathrm{F}_{1 \mathrm{r}}$ (reciprocal) hybrids $\mathrm{P}_{5} \times \mathrm{P}_{3}$ and $\mathrm{P}_{6} \times \mathrm{P}_{5}$ showed highly significant positive effects for the same trait. At the same time, the $F_{1 r}$ (reciprocal) hybrid $\mathrm{P}_{5} \times \mathrm{P}_{3}$ gave the highest value (1.000) for the same trait.

The $\mathrm{F}_{1}$ hybrids $\mathrm{P}_{2} \times \mathrm{P}_{3}$ and $\mathrm{P}_{6} \times \mathrm{P}_{7}$ gave the largest and significant values for F.D.cm of 0.551 and 0.694 , respectively. While, the $\mathrm{F}_{1 \mathrm{r}}$ (reciprocal) hybrid $\mathrm{P}_{6} \times \mathrm{P}_{2}$ gave significant value of 0.666 for the same trait.

For F.Sh.I., the $\mathrm{F}_{1}$ hybrid $\mathrm{P}_{2} \times \mathrm{P}_{6}$ gave highly significant value of 0.863 . At the same time, the $F_{1 \mathrm{r}}$ (reciprocal) hybrids $\mathrm{P}_{6} \times \mathrm{P}_{1}$ and $\mathrm{P}_{6} \times \mathrm{P}_{5}$ gave significant values of 0.698 and 0.433 for the same trait, respectively.

For T.S.S. $\%$, the $\mathrm{F}_{1}$ hybrids $\mathrm{P}_{2} \times \mathrm{P}_{3}$ and $\mathrm{P}_{4} \times \mathrm{P}_{7}$ gave highly significant values of 0.779 and 0.826 , respectively. At the same time, the $\mathrm{F}_{1 \mathrm{r}}$ (reciprocal) hybrids $\mathrm{P}_{6} \times \mathrm{P}_{2}$ and $\mathrm{P}_{6} \times \mathrm{P}_{5}$ gave values of 0.333 and 0.500 for the same trait, respectively.
For W.F.g., the $\mathrm{F}_{1}$ hybrids $\mathrm{P}_{1} \times \mathrm{P}_{6}$ and $\mathrm{P}_{2} \times \mathrm{P}_{3}$ gave highly significant values of 31.23 and 34.61. At the same time, the $\mathrm{F}_{1 \mathrm{r}}$ (reciprocal) hybrids $\mathrm{P}_{6} \times \mathrm{P}_{3}$ and $\mathrm{P}_{6} \times$ $\mathrm{P}_{5}$ gave highly significant values of 23.17 and 9.33 for the same trait.

For No.F./P., the $\mathrm{F}_{1}$ hybrids $\mathrm{P}_{6} \times \mathrm{P}_{3}$ and $\mathrm{P}_{6} \times \mathrm{P}_{5}$ gave highly significant values of 4.605 and 5.105, respectively. At the same time, the $F_{1 r}$ (reciprocal) hybrids $\mathrm{P}_{4} \times \mathrm{P}_{1}$ and $\mathrm{P}_{4} \times \mathrm{P}_{3}$ gave highly significant values for the same trait.

The $\mathrm{F}_{1}$ hybrids $\mathrm{P}_{1} \times \mathrm{P}_{6}$ and $\mathrm{P}_{2} \times \mathrm{P}_{3}$ gave highly significant values of 0.928 and 1.333 for F.Y./P.kg., respectively. At the same time, the $\mathrm{F}_{1 \mathrm{r}}$ (reciprocal) hybrids $\mathrm{P}_{5} \times \mathrm{P}_{3}$ and $\mathrm{P}_{6} \times \mathrm{P}_{3}$ gave unsignificant value of 0.333 for the same trait.

In general, the hybrids exhibited variable effects for SCA and therefore showed different mode of action in the manifestation of heterosis. At the same time, the results showed variable effect in the inheritance of the studied traits. These results were in agreement with the results that obtained by Al-Araby, (2010); Pradip et al., (2013) and Sanin et al., (2014). 


\section{REFERENCES}

Abd El-Hadi, A. H.; M. M. Zaghloul and A. H. Gabr (2004). Nature of gene action, heterosis and inbreeding depression of yield and yield component traits in squash (Cucurbita pepo, L.). Zagazig J. Agric, Res., 31(6): 2707-2725.

Abd El-Hadi, A. H.; Z. A. Kosba; Z. M. El-Diasty; El-S. H. Askar and G. M. Shamloul (2001). Evaluation of $F_{1}$ hybrids among new selected inbred lines of sweet melon (Cucumis melo var. aegyptiacus, L.).J. Agric. Sci., Mansoura Univ., 26(5): 2831-2845.

Abdein, M. A. (2005). Quantitative genetics of some economic traits in squash (Cucurbita pepo. L). M. Sc. Thesis, Fac. of Agric., Mansoura Univ. Egypt.

Al-Araby, A. A. (2010). Estimation of heterosis, combining ability and heritability in inter varietals crosses of summer squash (Cucurbita pepo L.) .Ph. D. Thesis, Fac. of Agric., Tanta Univ. Egypt.

Al-Ballat, I. A. (2008). Breeding studies on summer squash crop (Cucurbita pepo L.). M. Sc. Thesis, Fac. of Agric., Tanta Univ. Egypt.

Ana, I. Lopez-Sese and J. Staub (2002). Combining ability analysis of yield components in cucumber. J. Amer. Soc. Hort. Sci. 127(6): 931-937.

Cockerham, C. C. (1963). Estimation of genetic variances. Statistical Genetics and Plant Breeding. NAS-NRC, 982, pp.53-68.

Douglas, W. N.; W. R. Maluf; A. R. Figueira; G. M. Maciel; L. A. Gomes and C. A. Benavente (2011). Combining ability of summer-squash lines with different degrees of parthenocarpy and PRSV-W resistance. Genetics and Molecular Biology, 34 (4): 616-623.

El-Adl, A. M; Z. A. Kosba; Z. M. El-Diasty and A. H. Abd El-Hadi (1996). Types of gene action associated with the performances of hybrids among newly developed inbred lines of agoor, (Cucumis melo var. chate, L.). J. Agric. Sci. Mansoura Univ., 21(8): 2821-2835.

El-Sharkawy, Gehan A. M. (2000). An analytical study for the genetic behavior of some important characters of summer squash (Cucurbita pepo,L.) using a diallel cross system among seven inbred lines of "Eskandrani" cultivar. M. Sc. Thesis, Fac. of Agric., Alex. Univ. Egypt.

Feyzian, E.; H. Dehghani; A. M. Rezai and M. J. Javaran (2009). Diallel cross analysis for maturity and yieldrelated traits in melon (Cucumis melo, L.). Euphytica, 168(7):215-223.

Gabr, A. H. (2003). Nature of gene action and performance of hybrids in squash (Cucurbita pepo, L.). M.Sc. Thesis, Fac. of Agric., Mansoura Univ. Egypt.
Griffing, B. (1956). Concept of general and specific combining ability in relation to diallel crosses system. Aust. J. Biol. Sci., 9:pp 463-493.

Helmy, E. M. S. (1993). Development of pure lines of summer squash and their use in hybrid production. J. Agric. Sci. Mansoura Univ., 18(2): 489-498.

Iathet, C. and K. Piluek (2006). Heritability, heterosis and correlations of fruit characters and yield in Thai slicing melon (Cucumis melo L. var. conomon makino). Kasetsart Journal, Natural Sciences, 40(6):20-25.

Jahan, T. A.; A. K. M. Islam; M. G. Rasul; M. A. K. Mian and M. M. Haque (2012). Heterosis of qualitative and quantitative characters in sweet gourd (Cucurbita moschata Duch.ex Poir). African Journal of Food, Agriculture, Nutrition and Development, 12(3): 61866199.

Kamooh, A. A. (2002). Studies on general and specific combining abilities of some characters in squash (Cucurbita pepo, L.). J. Agric. Sci. Mansoura Univ., 27(7): 4861-4888.

Marie, A. K.; M. Y. Moualla and M. G. Boras (2012). Heterosis study of some quantity characters of squash (Cucurbita pepo, L.). Damascus J. of Agric. Sci., 28(1):339-354.

Matzinger, D. F. and O. Kempthorne (1956). The modified diallel Table with partial inbreeding and interactions with environment. Genetics, 41(1): 822-833.

Mishra, G.; J. R. Yadav; N. S. Parihar; J. K. Yadav; S. Kumar and Y. Alok (2007). Study on heritability and genetic advance in cucumber (Cucumis sativus, L.). Progressive Research 2(3):187-189.

Pradip, K.; A. D. Munshi; T. K. Behera; R. Kumar; C. Kaur and B. K. Singh (2013). Hermaphrodite inbreds with better combining ability improve antioxidant properties in ridge gourd [Luffa acutangula (Roxb.) L.]. Euphytica, 191(3):75-84.

Sanin, O. G.; L. V. B. Burbano; G. A. O. Narvaez; M. P. V. Restrepo; D. B. Garcia and F. A. V. Cabrera (2014). Inbreeding and gene action in butternut squash (Cucurbita moschata) seed starch content. Rev. Fac. Nal. Agr. Medellin, 67(1): 7169-7175.

Steel, G. D. and H. Torrie (1960). Principles and procedures of statistics. Mc. raw. Hill Book Company, INC, New York, pp 43.

Thangamani, C.; L. Pugalendhi; T. Sumathi; C. Kavitha and V. Rajashree (2011). Estimation of combining ability and heterosis for yield and quality characters in bitter gourd (Momordica charantia L.). Electronic Journal of Plant Breeding, 


\section{الملخص العري}

\section{قوة الهجن وللسلوك الوراثي لبضن صفلت المحصط ومكوناته فيقرع الكوسة}

أثشرف هسين عبد الهادي، عله ماهر العل، حورية محمد فتهي ومحمد عبد الحميد عابين

لُٔلهرت النتائج وجود قيما معنوية لقوة الهجين قيلساً من

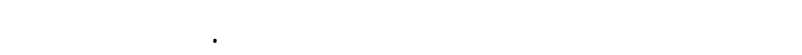

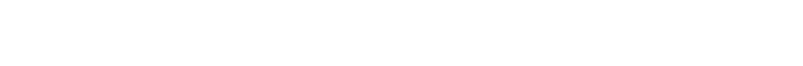

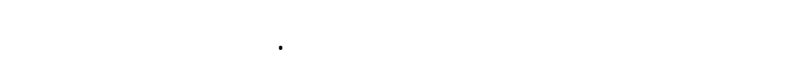

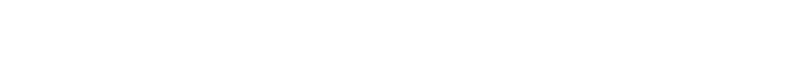

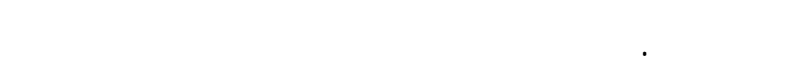

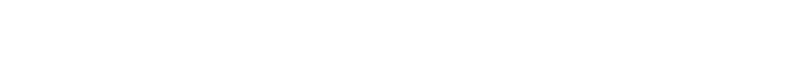

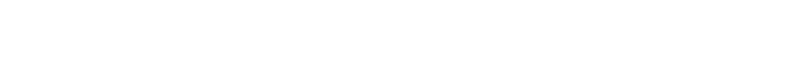

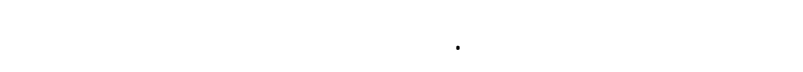
(الأب الأول)، Eskandarani

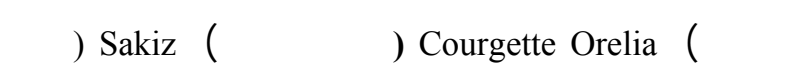

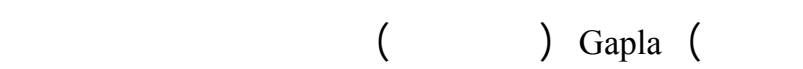
طول الثمرة (بلللسنتيمتر)، دللـشكل الثمرة، عدد الثمار للكل

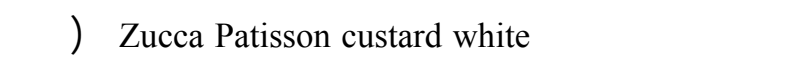
الثاني) أفضل مصدر لصفة النبة المئوية للسكريت الذائبة

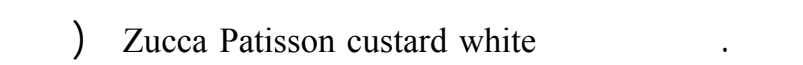

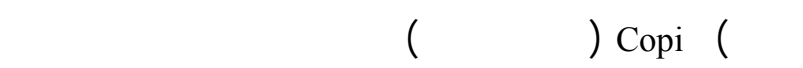
الثمرة (بللسنتيمتر) ومتونط وزن الثمرة (بالجرلم).

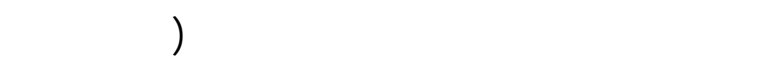

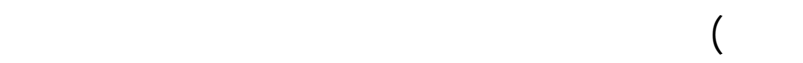

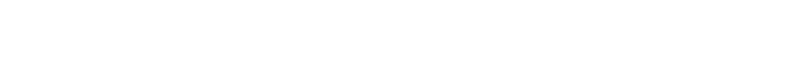
مكررات وقد إجريت هذه الدرلسة في مزرعة قها البحثي

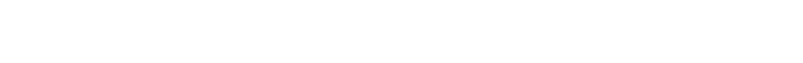
البحوث الزراعية بالجيزة بجمهورية مصر العربية.
في هذه الدرلسة قم ققيم الاباء والهجن النانجة (الهج ـن والهجن العكسية) من لمستخدلم ظطم التهجين الدوري الكلمل

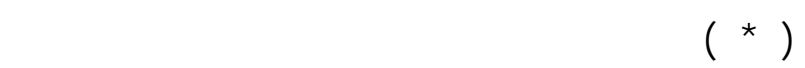
من قوة الهجين وللسلوك الوراثي لبعض صفات المحصول ومكوناته في قرع الكوسة.

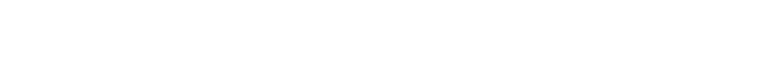

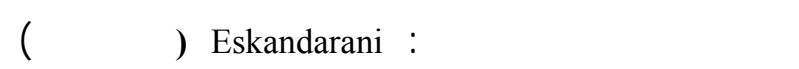
All Green (الأب الثاني) Zucca Patisson custard white

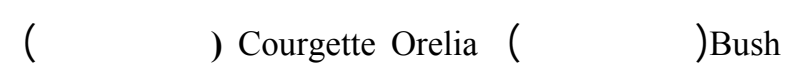
Gapla، الأب الخ Lمس)، Copi (الأب اللس اسل)،

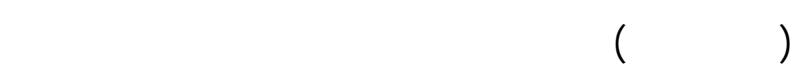

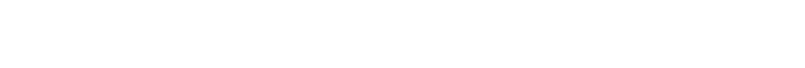
جمهورية مصر العربية، والأب الثاني من فرنسا والثال اث الثالي

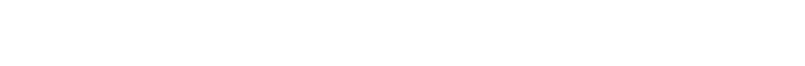

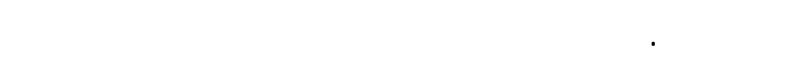

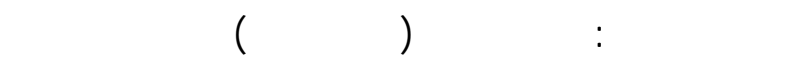

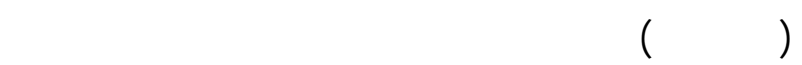

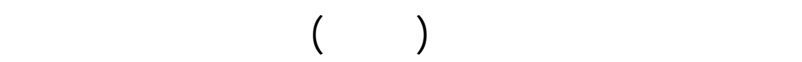

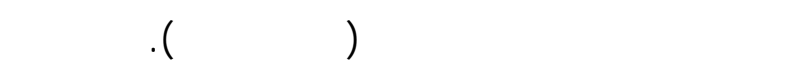
النتائج وجود إختلافلت كبيرة بين هجن الجيل الأول والأل الأول

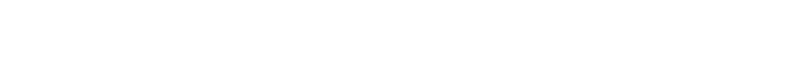

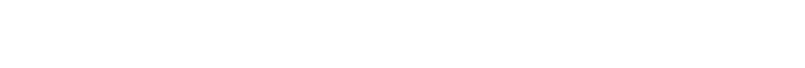

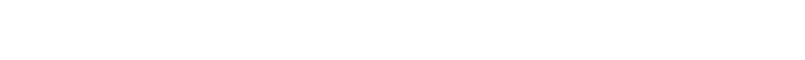

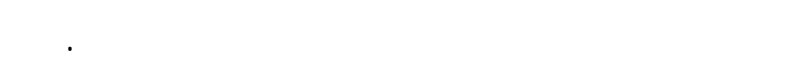

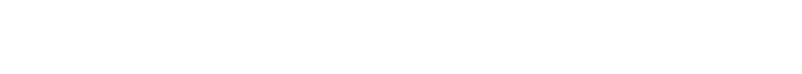
المحصول ومكوناته مطل الدرلمة. 

